\title{
Optimization Algorithm and Realization of the Phase Frequency Characteristics of Passive Network
}

\author{
Meifang Cai \\ Nanchang Institute of Science \& Technology
}

\begin{abstract}
Keywords: Amplitude-frequency Characteristics; Phase Frequency Characteristics; Group Delay; Optimization
\end{abstract}

\begin{abstract}
Passive network is a critical component which is commonly used in electronic and communication device. With the development of digital communication, the demands to the phase frequency characteristics of passive network have become higher and higher, while there is contradiction between the phase frequency characteristics and the amplitude-frequency characteristics. How to get a better group delay characteristics from passband and to gain a larger attenuation from stopband has been the research topic for the relevant national scientists to explore and study. The main solution in the past mainly starts from the network theory
\end{abstract}

After decades of efforts, the technology has matured, but progressing is very slow. This paper proposes a method that the optimization of passive network element parameters can make the frequency of a phase curve approximate a straight line. The method is firstly to determine a straight line on a phase frequency coordinates as the initial value of the ideal curve. Then to optimize component parameters, making the phase curve approximate this line and to obtain a new element value.

\section{Introduction}

With the development of digital communications, the demands to frequency characteristics of passive networks have become higher and higher. But there is a contradiction between the amplitude-frequency characteristics and phase frequency characteristics. How to gain a better group delay characteristic from passband and to gain a larger attenuation from stopband has been the research topics for relevant national scientists to explore and study. So far, the main way to solve this problem is to start from the study of the traditional network theory. For example, make the filter approximate phase function in passband and approximate the function with good frequency characteristics in stopband. Delay equalizer can be connected to the filter with a good frequency characteristics. The former is difficult to achieve an optimal compromise, since both passband delay and stopband attenuation are not at their best. For the latter, the circuit is too complex that brings many difficulties in manufacture.

The research uses circuit optimization technology instead of traditional network design to optimize comprehensive phase and frequency characteristics and the amplitude-frequency characteristics of passive network simultaneously.

\section{Group Delay}

The signal transmitted in an electronic device generally has a certain band-width, that means it contains many frequency components. To transmit such signals undistorted, in addition to make the filter have a certain bandwidth and to make the amplitude of each frequency in the signal be transmitted with certain ratio (i.e. no frequency distortion is allowed in transmission), the transmission speed of each frequency component shall also be the same (i.e. no phase distortion is allowed in transmission), so that the output terminal can restore the original signal.

We know that the phase delay between the phase and angular frequency have the following relationship:

$\tau_{p}=\varphi \div \varpi$ 


$$
\begin{aligned}
& \tau_{p}-\text { phase delay } \\
& \varphi-- \text { phase } \\
& \omega-\_ \text {angular frequency }
\end{aligned}
$$

For not generate phase distortion, it is required phase characteristic is linear, as shown in Fig 1 (a). In addition, the concept is also commonly used to group delay. Then

$$
\tau_{d}=\frac{d \varphi}{d \omega}
$$

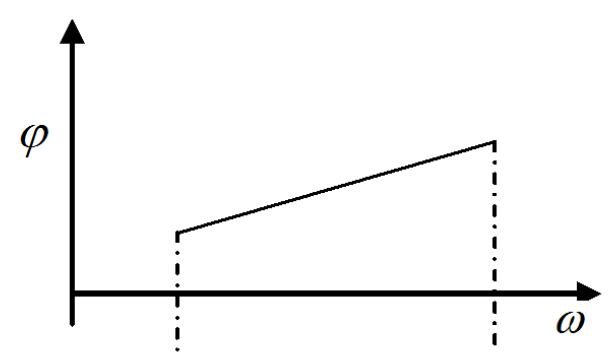

Figure 1. (a) Phase characteristics without phase distortion

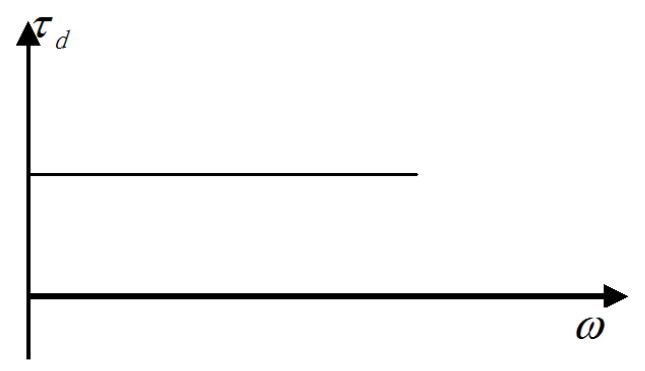

Figure 1. (b) Group delay characteristics without phase distortion

$\tau_{d}$ means group delay, obviously, in terms of the group delay $\tau_{d}$, no phase distortion condition is:

$$
\tau_{d}=\text { constant }
$$

In the electronic device which transmits a single frequency signals, always use the concept of phase delay. The electronic device which has a transmission bandwidth of the signal, then always use the concept of group delay. Usually refer group delay characteristic as delay characteristics.

\section{The Establishment of the Objective Function in Optimization Phase Frequency Characteristics}

In the early 1970s, circuit optimization technology was produced that can optimize amplitude-frequency characteristics in network. This technology has been continuously improved and perfected. It can solve the problems in passive network design which are needed to be solved in modern communication technology but cannot be solved by the conventional theoretical approach.

Use circuit optimization technology instead of traditional network design to optimize phase frequency characteristics of passive network simultaneously. While it had done a lot of preparatory work before, the amplitude-frequency characteristics of optimization technology was closed to mature. The method of presentation is divided into three steps.

The first step: establish the objective function.

The second step: find out the gradient of the objective function.

The third step: improve the modern optimization methods to obtain the best network element 
parameter values.

Establish Objective Function for This Filter Network Optimization. The optimization of amplitude-frequency characteristics are worked at each frequency point separately and then summed, general objective function can be written as:

$$
\begin{aligned}
& F_{1}(X)=\frac{1}{2} \sum_{k=1}^{\rho} W\left(\omega_{k}\right)\left[\left|V_{0}\left(X, \omega_{k}\right)\right|-\mid \tilde{V}_{0}\left(\omega_{k}\right)\right]^{2} \\
& V_{0}=V_{0}\left(X, \omega_{k}\right)=a\left(X, \omega_{k}\right)+j b\left(X, \omega_{k}\right)=a+j b \\
& X=\left(x_{1}, x_{2}, x_{3} \cdots, x_{n}\right) \text { is the component parameters, also design variables, } \omega_{k} \text { represents }
\end{aligned}
$$
the frequency of sampling points, $W\left(\omega_{k}\right)$ is a weighting function of frequency on the each sampling point $\omega_{1}, \omega_{2}, \omega_{3}, \cdots \omega_{\rho}, \quad \tilde{V}_{0}\left(\omega_{k}\right)$ is the ideal value of the output voltage.

Generally, amplitude-frequency characteristic and phase frequency characteristics need to be optimized at the same time, by weight function considered. The objective function is:

$$
F(X)=\frac{1}{2} \sum_{k=1}^{p} 1(x)\left[V\left(X, \omega_{k} \mid\right)-\mid \tilde{V}_{0}\left(\omega_{k}\right)\right]^{2}+\frac{1}{2} \sum_{k=1}^{p} W_{2}(\omega)\left|\varphi\left(X, \omega_{k}\right)-\tilde{\varphi}_{0} \phi_{k}\right|^{2},
$$

Wherein, $W_{1}\left(\omega_{k}\right)$ is the right of frequency characteristic on amplitude of each frequency point, $W_{2}\left(\omega_{k}\right)$ is the right of phase frequency characteristics on amplitude of each frequency point.

\section{The Gradient of the Objective Function in Phase Frequency Characteristics}

Higher order nonlinear function, the most effective method to optimize such function is Simulate Newton method (analytical method, gradient method), including conjugate gradient method, D and BFGS and so on. The key of taking advantage of these gradient method (not spend a lot of time, which is the minimum amount) is to obtain objective function gradient component parameters accurately and effectively.

The i element gradient of the objective function is:

$$
\begin{aligned}
& \frac{\partial F_{2}}{\partial x_{1}}=\sum_{k=1}^{\rho} W\left(\omega_{k}\right)\left[\omega_{v}\left(X, \omega_{k}\right)-\tilde{\varphi}_{v}\left(\omega_{k}\right)\right] \frac{\partial \varphi_{v}}{\partial x_{1}} \\
& \text { By the above, we have: } \frac{\partial \varphi_{v}}{\partial x_{1}}=\frac{\partial \operatorname{arctg} \frac{b}{a}}{\partial x_{1}}=\frac{a \frac{\partial b}{\partial x_{1}}-b \frac{\partial a}{\partial x_{1}}}{a^{2}+b^{2}}
\end{aligned}
$$

$\frac{\partial a}{\partial x_{1}}$ and $\frac{\partial b}{\partial x_{1}}$ are the derivatives of the element parameters, which are the real and imaginary parts of the output voltage, they are sensitivities. So only determine the sensitivity that can be done to the gradient of the objective function solving. Sensitivity analysis is bound to encounter problems in network design, while as a direct calculation of the sensitivity, the workload shall be very large. How to simplify these calculations is a great concerned problem to network design workers.

\section{Conclusion}

The rapid development of modern digital communication requires filters to have better group delay characteristics in passband and steep attenuation in stopband, while there is a contradiction between phase-frequency characteristics and amplitude-frequency characteristics, so it is very difficult. The relevant scientists of different countries have done a lot of work, but so far the results are unsatisfactory. This paper puts circuit optimization technology into the design of filter, and proposes the method of optimizing the amplitude-frequency characteristics and phase-frequency 
characteristics at the same time. It is not the traditional improvement method, but is a new one. This method allows the filter in the amplitude-frequency characteristics and phase-frequency characteristics are of a reasonable compromise to achieve the best conditions for each state. This reasonable compromise is proposed by the system designers based on overall performance requirements, which can be freely and easily achieved through the mediation right functions, which cannot be achieved by the traditional method. So the proposed method in the actual design and the way of manufacturing filter has obvious advantages comparing to the traditional method, which can be clearly seen from the examples in this paper.

\section{References}

[1] Branson P. Electronics and communications technology in society. The Daytona racetrack [J]. Electronics Education, 2003, 2003:17-20.

[2] Branson P. Electronics and communications technology in society. Traffic cameras in Manchester [J]. Electronics Education, 2002, 2002.

[3] Martin J, Branson P. Progression in electronics and communications technology [J]. (C) data, 2002.

[4] Kumashiro S. Simulation Technology of CMOS Process and Device (Special Issue on Simulation Technology for Electronics and Communications) [J]. Journal of the Institute of Electronics Information \& Communication Engineers, 2000, 83.

[5] Nishi K. Three-dimensional Process Simulation ( Special Issue on Simulation Technology for Electronics and Communications)[J]. Journal of the Institute of Electronics Information \& Communication Engineers, 2000, 83(11):833-837.

[6] Yamashita E. Simulation Engineering as Integrated Technologies (Special Issue on Simulation Technology for Electronics and Communications)[J]. Journal of the Institute of Electronics Information \& Communication Engineers, 2000, 83:808-812.

[7] Rughooputh S D, Rughooputh H C S. Polymers for electronics and communications[C]// Africon, 1999 IEEE. IEEE, 1999:55-60 vol.1.

[8] Thompson J. Open Forum of the Electronics and Communications Division, Thursday 1st July 1999[J]. Electronics \& Communication Engineering Journal, 1999, 11(2):66-66.

[9] Taylor J. Technology Foresight: IT, Electronics and Communications [J]. Electronics \& Communication Engineering Journal, 1995, 7(6):234-234.

[10] Allen J, Berker A N, Birgeneau R, et al. Basic and applied research in the field of electronics and communications [J]. Basic \& Applied Research in the Field of Electronics \& Communications, 1989.

[11]Laybourn P J R. Optical Fibre Electronics and Communications [J]. IEE review, 1982, 28(10):709-709.

[12] Takanami, Itsuo, Honda, et al. A characterization of Parikh's theorem and the semilinear sets by commutative semigroups with length. [J]. Electron.commun.japan, 1969(10):179-184. 